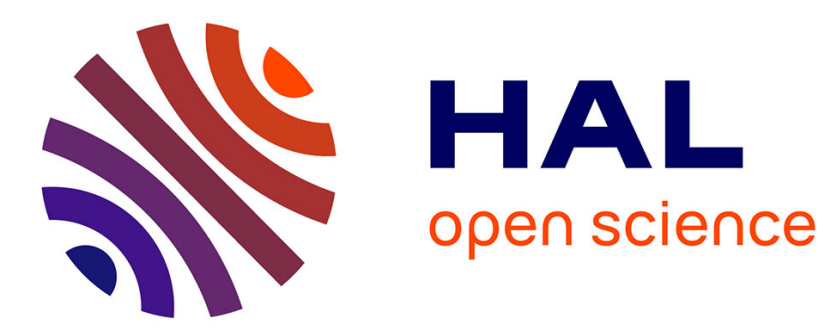

\title{
Caractérisation par pompage optique d'un super réseau à courte période GaAs/AlAs
}

\author{
G. Bacquet, J. Bandet, F. Fabre, J. Frandon, R. Taouint, R. Planel
}

\section{To cite this version:}

G. Bacquet, J. Bandet, F. Fabre, J. Frandon, R. Taouint, et al.. Caractérisation par pompage optique d'un super réseau à courte période GaAs/AlAs. Revue de Physique Appliquée, 1989, 24 (3), pp.337342. 10.1051/rphysap:01989002403033700 . jpa-00246055

\section{HAL Id: jpa-00246055 https://hal.science/jpa-00246055}

Submitted on 1 Jan 1989

HAL is a multi-disciplinary open access archive for the deposit and dissemination of scientific research documents, whether they are published or not. The documents may come from teaching and research institutions in France or abroad, or from public or private research centers.
L'archive ouverte pluridisciplinaire HAL, est destinée au dépôt et à la diffusion de documents scientifiques de niveau recherche, publiés ou non, émanant des établissements d'enseignement et de recherche français ou étrangers, des laboratoires publics ou privés. 


\title{
Caractérisation par pompage optique d'un super réseau à courte période GaAs/AlAs
}

\author{
G. Bacquet $\left({ }^{(}\right)$, J. Bandet $\left({ }^{1}\right)$, F. Fabre $\left({ }^{1}\right)$, J. Frandon $\left({ }^{1}\right)$, R. Taouint $\left({ }^{1}\right)$ et R. Planel $\left({ }^{2}\right)$ \\ (1) Laboratoire de Physique des Solides, Associé au CNRS/UA74, Université Paul Sabatier, 31062 Toulouse \\ Cedex, France \\ (2) Laboratoire de Microstructure et de Microélectronique/LP20 CNRS, 196 Avenue Henri Ravera, \\ 92220 Bagneux, France
}

(Reçu le $1^{\text {er }}$ juillet 1988, révisé le 20 octobre 1988, accepté le 29 novembre 1988)

\begin{abstract}
Résumé. - On a utilisé les techniques du pompage optique pour étudier les propriétés de la luminescence d'un super réseau à courte période GaAs/AlAs. Le spectre d'excitation de la luminescence montre, outre les transitions bien connues $1 \mathrm{e} \rightarrow \mathrm{hh}$ et $1 \mathrm{e} \rightarrow \ell \mathrm{h}$, des oscillations de relaxation observables en raison de la présence de puits élargis dans l'échantillon. Quelle que soit la longueur d'onde excitatrice, la polarisation de la raie excitonique principale décroît avec l'application d'un champ magnétique transverse. Les valeurs du temps de relaxation de spin et du temps de vie des électrons photocréés ont été estimées respectivement à $1,1 \times 10^{-10}$ et $1,7 \times 10^{-10} \mathrm{~s}$. Le collage de l'échantillon sur le support crée des contraintes incontrôlées auxquelles les résultats sont très sensibles.
\end{abstract}

\begin{abstract}
Optical pumping techniques are used to study the luminescent properties of a GaAs / AlAs shortperiod superlattice. The luminescence excitation spectrum, aside the well-known $1 \mathrm{e} \rightarrow \mathrm{hh}$ and $1 \mathrm{e} \rightarrow$ $\ell_{h}$ transitions, exhibits relaxation oscillations caused by the presence of enlarged wells in the sample. The polarization of the prevailing excitonic luminescence line is magnetic field dependent whatever the exciting wavelength. The determined values of the spin relaxation time and of the lifetime of the photogenerated electrons were respectively $1.1 \times 10^{-10}$ and $1.7 \times 10^{-10} \mathrm{~s}$. Small uncontrolled strains, due to the fixation of the sample on the holder may strongly affect the results.
\end{abstract}

La maîtrise de l'épitaxie par jet moléculaire permet la fabrication de nouvelles microstructures semiconductrices. Les puits quantiques $\mathrm{GaAs} / \mathrm{Ga}_{1-x} \mathrm{Al}_{x} \mathrm{As}$ avec $x<0,4$ ont été particulièrement étudiés.

Ultérieurement il est apparu que les super réseaux $(\mathrm{GaAs})_{m} /(\mathrm{AlAs})_{n}$ constituaient des hétérostructures permettant un grand éventail d'études tant expérimentales que théoriques. En effet, dans ce cas, le matériau barrière $\mathrm{AlAs}$ est à bande interdite indirecte.

Dans les deux dernières années, plusieurs études de ces «pseudo alliages » ont été menées [1-6]. A partir de mesures optiques, pour l'essentiel luminescence et spectre d'excitation de cette dernière, il ressort de ces travaux que l'on peut mettre en évidence des pseudo alliages à bande interdite indirecte, ou "pseudo-directe », pour des périodes $P=\ell_{\mathrm{GaAs}}+\ell_{\mathrm{AlAs}}$ suffisamment courtes et des concentrations d'AlAs $\bar{x}=\ell_{\mathrm{AlAs}} / P$ suffisamment élevées [3-6]. Les résultats expérimentaux ont été convenablement interprétés à l'aide d'une théorie où les états électroniques et les niveaux d'énergie sont calculés dans l'approximation de la fonction enveloppe [7] appliquée au voisinage des extrema de bandes situés aux points $\Gamma$, $\mathrm{X}$ et $\mathrm{L}$ de la zone de Brillouin de chacun des matériaux constitutifs. Cela a permis à Danan et al. $[3,6]$ de déduire très précisément le paramètre de décalage de bandes au point $\Gamma, \Delta \equiv\left[\Delta E_{\mathrm{c}}^{\Gamma} / \Delta E_{\mathrm{g}}\right]=0,67$.

L'utilisation d'une lumière polarisée circulairement pour exciter la luminescence permet d'orienter les excitations élémentaires, et la mesure du degré de polarisation circulaire $\mathfrak{S}$ de la luminescence donne accès à la polarisation de spin à l'équilibre. Cela peut se révéler utile pour l'identification des transitions [8]. De telles mesures, menées en parallèle avec l'étude du spectre d'excitation de la luminescence (SEL) ont permis d'identifier sans ambi- 
guïté les transitions entre les premiers niveaux électroniques de la bande de conduction et les sousbandes de trous lourds et légers dans les structures $\mathrm{GaAs} / \mathrm{Ga}_{1-x} \mathrm{Al}_{x} \mathrm{As}$ [9-11].

La technique du pompage optique $[12,13]$ consiste à mesurer $\mathfrak{T}$ et à en étudier la variation en fonction d'un champ magnétique $\mathbf{B}$ appliqué perpendiculairement à la lumière excitatrice (effet Hanle). On peut ainsi mesurer expérimentalement deux quantités qui sont reliées à la durée de vie $\tau$ de l'électron photocréé dans la bande de conduction et au temps de relaxation de spin $T_{1}$. Dans le modèle simple d'une population d'électrons polarisée initialement $P_{\text {I }}$ par orientation optique et d'une population de trous non polarisée, le degré de polarisation de la lumière de luminescence est donné par :

$$
\mathfrak{T}=P_{\mathrm{I}} P_{\mathrm{R}}\left[T_{1} /\left(T_{1}+\tau\right)\right]
$$

où $P_{\mathrm{R}}$ serait le degré de polarisation de luminescence d'une population de spin complètement orientée. $P_{\mathrm{I}}$ et $P_{\mathrm{R}}$ dépendent des probabilités de transition entre les divers états de la bande de valence et ceux de la bande de conduction. Dans le cas des super réseaux, le confinement entraîne une séparation en énergie entre les sous-bandes de trous lourds et de trous légers. Il en résulte que la valeur de $P_{\mathrm{I}}$ peut changer suivant l'énergie d'excitation. Sur la figure 1 , on peut voir que pour une excitation $\sigma^{+}$résonnante à partir de la sous-bande des trous lourds, seuls les électrons de spin $-1 / 2$ sont créés dans la bande de conduction. Les électrons ne pouvant, à $1,7 \mathrm{~K}$, que se recombiner sur la sousbande de trous lourds (canal désigné par $\mathrm{A}$ ), $P_{\mathrm{I}} P_{\mathrm{R}}$ vaut donc 1 . Si les spins électroniques sont en même temps générés à partir de la sous-bande de trous légers, il y a aussi des électrons de spin $+1 / 2$ dans la bande de conduction. La recombinaison désignée par $\mathrm{D}$ est également possible et l'on trouve que $P_{\mathrm{I}} P_{\mathrm{R}}$ est alors inférieur à l'unité.

Si les probabilités de transition optique en absorption et en émission sont, pour les transitions trous lourds $\leftrightarrow$ électrons $(\mathrm{hh} \leftrightarrow 1 \mathrm{e})$ et trous légers $\rightarrow$ électrons $\left(\ell_{\mathrm{h}} \rightarrow 1 \mathrm{e}\right)$, dans le rapport des forces

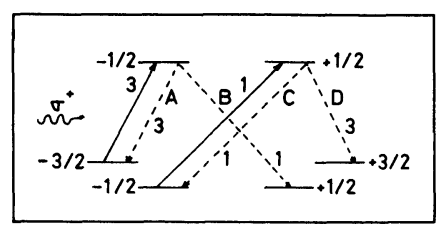

Fig. 1. - Absorption (trait plein) et émission (tirets) sous excitation avec de la lumière polarisée $\sigma^{+}$(nous indiquons les forces d'oscillateur relatives).

[Absorption (full lines) and emission (dashed line) upon $\sigma^{+}$polarized light (relative oscillator strengths are indicated).] d'oscillateurs, c'est-à-dire de 3 à 1 , on trouve que, pour une excitation polarisée circulairement $\sigma^{+}$, $P_{\mathrm{I}} P_{\mathrm{R}}=0,5$. En fait, si l'on tient compte des densités d'états conjointes, particulièrement piquées pour les trous légers du fait de la non-parabolicité des bandes, comme l'ont fait Twardowski et Hermann [14], il apparaît qu'à des énergies proches de la transition excitonique associée à l'excitation à partir des trous légers, le taux de création d'électrons de spin $+1 / 2$ est très fortement augmenté. Cela conduit à une diminution du degré de polarisation de la luminescence telle qu'il peut même prendre des valeurs négatives. Il faut bien faire remarquer qu'un tel accident est lié au fait qu'absorption et émission mettent en jeu des états de valence de symétries différentes.

Par ailleurs, la levée de dégénérescence entre trous lourds et légers ralentit probablement la relaxation de spin des trous, rendant hasardeuse la simplification, courante dans les semiconducteurs cubiques, d'une population de trous désorientée.

Enfin, la variation du degré de polarisation sous l'effet d'un champ magnétique transverse (effet Hanle) est caractérisée par une loi Lorentzienne

$$
\mathfrak{T}(\mathrm{B})=\mathfrak{T}(0) \frac{1}{1+\omega^{2} T_{1}^{* 2}}
$$

où $\omega$ est la fréquence de Larmor et $T_{1}^{*}$ la durée de vie de polarisation:

$$
T_{1}^{*-1}=T_{1}^{-1}+\tau^{-1} .
$$

Nous souhaitons, dans cette contribution, montrer que la technique du pompage optique appliquée à l'étude d'un super réseau à courte période GaAs / AlAs bien particulier, se révèle très utile pour sa caractérisation.

\section{Echantillon et appareillage.}

L'échantillon étudié a été obtenu en épitaxie par jet moléculaire au Laboratoire de Microstructures et de Microélectronique du CNRS à Bagneux. Il est caractérisé par $P=3,88 \mathrm{~nm}$ et $x=0,34$ déterminés par diffraction de rayons $X$. Suivant la classification de Danan et al. [3] cet échantillon est dit « de transition » en ce sens que les bandes de conduction issues de $\Gamma$ et $\mathrm{X}$ sont à peu près à la même énergie. Pour les recombinaisons entre les électrons issus de $\Gamma$ et les sous-bandes de trous lourds et légers le calcul donne respectivement $E_{\mathrm{hh}}^{\Gamma}=1,831 \mathrm{eV}$ et $E \Gamma_{\mathrm{h}}=$ $1,895 \mathrm{eV}$. Entre les électrons issus de $\mathrm{X}$ et les trous lourds, on trouve $E^{\mathrm{X}}=1,86 \mathrm{eV}$.

Dans notre montage expérimental, la luminescence est excitée à l'aide d'un laser à colorant pompé par un laser argon $4 \mathrm{~W}$. Le colorant utilisé (DCM) nous permet de faire varier la longueur d'onde excitatrice $\lambda_{\text {exc }}$ entre 610 et $690 \mathrm{~nm}\left(2,032 \geqslant h v_{\text {exc }} \geqslant\right.$ 
$1,796 \mathrm{eV})$. Le faisceau incident arrivant perpendiculairement au plan des couches est focalisé sur la surface en une tâche d'environ $300 \mu \mathrm{m}$ de diamètre. Les densités de puissance utilisées sont inférieures ou égales à $10 \mathrm{~W} . \mathrm{cm}^{-2}$. La luminescence est détectée à l'aide d'un monochromateur Jobin-Yvon HRS2 et d'un photomultiplicateur à cathode $\mathrm{GaAs}$ refroidi thermiquement. La détermination de :

$$
\mathfrak{S}=\left[\left(L_{+}-L_{-}\right) /\left(L_{+}+L_{-}\right)\right]
$$

où $L_{+}\left(L_{-}\right)$est l'intensité de la composante $\sigma^{+}\left(\sigma^{-}\right)$ de la lumière de recombinaison est effectuée à l'aide de la méthode standard précédemment décrite [15] qui consiste à mesurer séparément le dénominateur puis le numérateur de la relation (4). La modulation, nécessaire à l'utilisation d'une détection synchrone, se fait à l'excitation et à la fréquence de $50 \mathrm{kHz}$ pour s'affranchir de possibles effets nucléaires affectant la relaxation de la polarisation des électrons et l'effet Hanle [16].

Nous avons utilisé un cryostat à hélium liquide permettant de travailler à $1,7 \mathrm{~K}$. Les mesures à des températures supérieures à $5 \mathrm{~K}$ ont été effectuées à l'aide d'un cryostat à circulation d'hélium. Dans tous les cas, l'échantillon, dont la surface est de quelques $\mathrm{mm}^{2}$, est fixé sur le porte-objet au moyen d'un petit point de graisse à vide qui, au refroidissement, peut induire localement des contraintes comme nous le verrons plus loin.

\section{Résultats.}

Sauf dans quelques cas spécifiques, que nous préciserons alors, tous les résultats présentés ont été obtenus à $1,7 \mathrm{~K}$.

La figure 2 montre le spectre de luminescence obtenu avec cet échantillon en utilisant une densité de puissance de $1,4 \mathrm{~W} . \mathrm{cm}^{-2}$ et une énergie excitatrice $h \nu_{\text {exc }}$ égale à $1,828 \mathrm{eV}$. L'impact du faisceau laser se situe à l'opposé du point de collage, donc dans une zone supposée exempte de contraintes. Le pic de luminescence principal est attribué à la recombinaison excitonique [3]. Son intensité intégrée varie linéairement en fonction de la densité de puissance excitatrice $W_{\text {exc }}$. Un groupe de raies apparaît à plus basses énergies $(1,716-1,764 \mathrm{eV})$. La variation de l'intensité intégrée des deux types de raies, quand on change la température d'observation (Fig. 3), semble exclure l'hypothèse que les raies basses énergies soient associées au niveau $\mathrm{X}$. En effet, dans ce cas, on attendrait une moins bonne thermalisation à haute température, due surtout au raccourcissement des durées de vie, ce qui devrait favoriser les raies hautes énergies. C'est ce que l'on observe dans des échantillons de ce type à gap clairement indirect [3] pour lesquels calculs et expérience donnent $E^{\mathrm{x}}<E^{\Gamma}$. Par ailleurs, les écarts en énergie entre la raie principale et les diverses

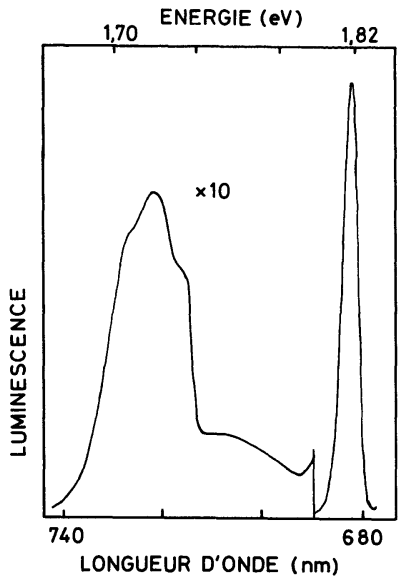

Fig. 2. - Spectre de luminescence enregistré à $1,7 \mathrm{~K}$ avec l'échantillon étudié. L'énergie excitatrice est de $1,828 \mathrm{eV}$ et la densité de puissance utilisée est de $1,4 \mathrm{~W} \cdot \mathrm{cm}^{-2}$.

[1.7 $\mathrm{K}$ luminescence spectrum recorded with the studied sample using a power density of $1.4 \mathrm{~W} . \mathrm{cm}^{-2}$. The exciting energy is $1.828 \mathrm{eV}$.]

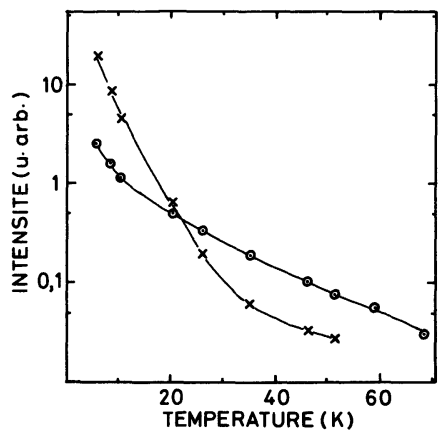

Fig. 3. - Variation en fonction de la température de l'intensité intégrée (approchée par le produit hauteur du signal $\times$ largeur à mi-hauteur) de la raie excitonique (x) et du groupe de raies apparaissant à basse énergie (0).

[Temperature dependence of the integrated line intensities (approximated by the peak photoluminescence signal times the full width of the spectrum at one-half the peak signal). Excitonic line (x) and low energy lines (0).]

composantes du groupe de raies apparaissant aux faibles énergies sur le spectre de luminescence ( $\delta E$ compris entre 64 et $112,2 \mathrm{meV}$ ) sont nettement supérieurs à ceux cités par Jiang et al. [17] et Danan [6] pour les échantillons à caractère $\mathrm{X}$ dominant.

La figure 4 montre le SEL obtenu en se calant à mi-hauteur du flanc basse énergie de la raie de luminescence principale (courbe en trait plein). Cinq pics désignés par A ... E sont clairement résolus. La distance entre les pics A $(1,8184 \mathrm{eV})$ et B $(1,8507 \mathrm{eV})$ est de $32,3 \mathrm{meV}$, les écarts $\mathrm{CD}$ et $\mathrm{DE}$ étant identiques et égaux à $44 \mathrm{meV}$.

Le spectre d'excitation de la polarisation est aussi représenté sur la figure 4 (courbe en pointillés). On 


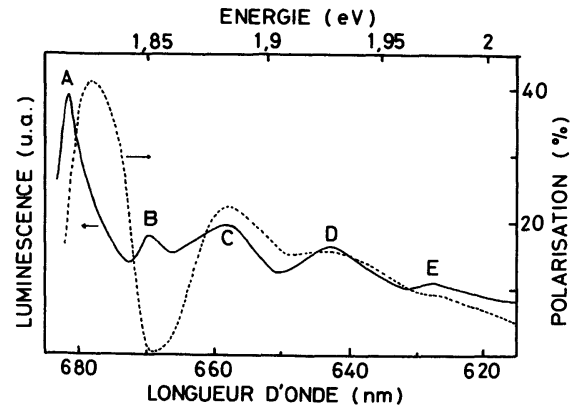

Fig. 4. - Spectres d'excitation de la luminescence (trait plein) et de la polarisation (tirets). Les énergies d'observation sont respectivement de 1,809 et $1,817 \mathrm{eV}$.

[Luminescence (full line) and polarization (dashed line) excitation spectra. The respective observation energies are 1.809 and $1.817 \mathrm{eV}$.

observe un maximum de polarisation de l'ordre de $40 \%$ à $1,827 \mathrm{eV}$ et un minimum presque nul à $1,853 \mathrm{eV}$. Chacun de ces deux extremums est décalé vers les grandes énergies par rapport aux pics $\mathrm{A}$ ou B. Les trois maximums de polarisation secondaires correspondent presque en énergie aux pics $C$, D et E. La nature de ces pics sera discutée ultérieurement.

Pour toutes les énergies excitatrices, si l'on excepte le court intervalle $1,845-1,857 \mathrm{eV}$, où la valeur de $\mathcal{T}$ est proche de 0 , la variation $\mathcal{T}(\mathbf{B})$ est Lorentzienne. Cela permet de mesurer une largeur Hanle $\Delta B$ dont la valeur, comprise entre $335 \mathrm{mT}\left(h v_{\text {exc }}=1,998 \mathrm{eV}\right)$ et $420 \mathrm{mT}\left(h v_{\text {exc }}=1,847 \mathrm{eV}\right)$ varie peu. Pour la région d'énergies situées autour de la raie $B$, les effets Hanle enregistrés peuvent être qualifiés d'anormaux. En effet, la valeur de $\mathfrak{T}(\mathbf{B})$ augmente légèrement, passe par un maximum puis décroît, rendant impossible toute détermination d'une largeur Hanle.

\section{Discussion.}

Le pic A du SEL (1,8184 eV) est interprété comme étant l'état fondamental de l'exciton formé à partir d'une paire électron-trou lourd $(1 \mathrm{e}-1 \mathrm{hh})$. Nous nous fondons surtout sur la très forte polarisation circulaire mesurée $\left(\mathfrak{T} \sim 40 \%\right.$ pour $h v_{\text {exc }}=$ $1,828 \mathrm{eV}$ ) qui implique peu de relaxation au sens large (relaxation de spin et d'énergie étant le plus souvent liées). A noter aussi la très faible valeur du décalage de Stokes $(2,4 \mathrm{meV})$.

Bien que l'énergie du pic $B(1,8505 \mathrm{eV})$ soit proche de la valeur $E^{\mathrm{x}}=1,86 \mathrm{eV}$ calculée, nous le faisons correspondre à l'excitation de la sous-bande des trous légers $(1 \mathrm{e}-1 \ell \mathrm{h})$. Une telle interprétation découle de la forte intensité de ce pic et de la quasiannulation de la valeur de $\mathfrak{T}$ pour $h v_{\text {exc }}=1,854 \mathrm{eV}$. En effet, la transition correspondant au pic B du SEL doit prendre en compte des trous de moment angulaire total différent de celui des trous lourds qui, eux, sont les seuls à être impliqués dans la recombinaison.

Les pics désignés par C, D et $\mathrm{E}$ (Fig. 4) sont observés respectivement à 1,$884 ; 1,928$ et $1,972 \mathrm{eV}$. Les calculs [6] montrant que la séparation énergétique entre les niveaux $1 \mathrm{e}$ et $2 \mathrm{e}$ atteint $500 \mathrm{meV}$ et que celle entre les niveaux $1 \mathrm{hh}$ et $2 \mathrm{hh}$ est de $125 \mathrm{meV}$, ces pics ne peuvent correspondre à l'excitation des états $n=2$ des électrons et des trous. Par ailleurs, chacun d'eux correspond à un maximum secondaire dans le spectre d'excitation de la polarisation. Ce fait, et l'écart identique entre $C, D$ et $E$, suggèrent de les attribuer à des " oscillations de relaxation". On sait que les électrons créés avec une énergie cinétique de valeur $n \hbar \omega_{\text {LO }}$ (où $n$ est entier et $\hbar \omega_{\text {LO }}$ désigne l'énergie d'un phonon optique longitudinal) relaxent le plus vite vers le bas de bande de? conduction car ce processus de perte d'énergie est beaucoup plus efficace que la diffusion par phonons acoustiques. Il en résulte un meilleur rendement de la luminescence et donc des oscillations de ce rendement.

Par ailleurs, la polarisation à l'équilibre dépend des processus de relaxation de spin électronique qui sont eux-mêmes liés étroitement à la relaxation de l'énergie. Cela est particulièrement vrai si, comme l'indiquent Twardowski et Hermann [14], le processus D'yakonov-Perel' [18] est dominant dans ces super réseaux. L'écart entre les maximums vaut alors, dans l'approximation des bandes paraboliques, $n \hbar \omega_{\text {LO }}\left[1+\left(m_{\mathrm{c}}^{*} / m_{\mathrm{h}}^{*}\right)\right]$. Ce phénomène a surtout été observé dans les semiconducteurs massifs II-VI, par exemple dans CdTe [19] où l'interaction entre électrons et phonons LO est plus efficace que dans les composés III-V. Dans le cas particulier du super réseau GaAs / AlAs que nous étudions, l'observation de ces "Oscillations de relaxation " est rendue possible, vraisemblablement par l'existence de puits élargis ayant pour origine un mauvais fonctionnement des caches du bâti d'épitaxie. Cette anomalie est bien sûr suggérée par d'autres expériences, structurales cette fois, mais nous l'ignorons volontairement dans cette discussion qui cherche à montrer ce qu'on peut tirer des techniques d'excitation de la luminescence en lumière polarisée circulairement. Ces puits élargis raccourcissent la durée de vie des états excités du super réseau et rendent ainsi la population d'équilibre, en bas de bande de conduction, plus sensible au détail de la relaxation d'énergie et de spin. Leur présence doit être révélée en luminescence. Ce sont eux, pensons-nous, qui sont à l'origine du groupe de raies à basse énergie évoqué plus haut. Sa dépendance en température peut alors s'expliquer, qualitativement, par l'augmentation du transport perpendiculaire aux couches [20]. La période d'oscillation que nous avons mesurée (44 meV) est compatible avec celle $(41 \mathrm{meV})$ que 
l'on peut calculer à partir des énergies du phonon LO et des masses effectives d'électrons et de trous lourds dans GaAs massif. L'énergie de base de cette cascade, celle de la raie C diminuée de $44 \mathrm{meV}$, tombe à vrai dire entre les raies $\mathrm{A}$ et $\mathrm{B}$. Cela laisse ouverte la question de la participation des trous légers à ces processus, des masses moyennes à considérer et de leur variation en énergie.

Nous avons signalé précédemment que la largeur Hanle ne variait que légèrement avec l'énergie d'excitation, sauf dans la région du pic B. Cela implique que l'essentiel de la précession de spin électronique se fasse dans l'état fondamental et donc que la durée de vie totale des excitons soit essentiellement celle de ce même état. La relaxation de spin dans cet état conduit à un taux de polarisation de la luminescence notablement inférieur à $100 \%$. Notons par ailleurs que l'effet Hanle est mesurable, et de valeur comparable, y compris lorsque des excitons lourds sont créés de façon résonnante (pic $\mathrm{A}$ du SEL). Cela fournit une preuve que l'exciton peut subir la précession Hanle dans cet état. La situation est donc très différente de celle des matériaux uniaxe, tels que $\mathrm{CdS}$, où la levée de dégénérescence des trous bloque l'effet Hanle [21].

L'exploitation tant qualitative que quantitative du phénomène observé nécessiterait la connaissance précise du rapport gyromagnétique de l'électron et du trou liés par interaction d'échange, et non pas du seul électron comme c'est le cas lorsque l'on suppose la population de trous libres complètement dépolarisée de spin. Or, une telle évaluation est difficile dans les super réseaux, notamment à cause de la levée de dégénérescence entre trous lourds et légers.

Pour évaluer $T_{1}$ et $\tau$, nous avons pris, comme l'ont fait Miller et al. [9], $g^{*}=-0,44$ qui est la valeur du rapport gyromagnétique de l'électron libre dans GaAs cristallin [22]. Lorsque seuls les trous lourds sont excités nous avons trouvé, pour $h v_{\text {exc }}=$ $1,828 \mathrm{eV}, \mathfrak{T}=0,40$ et $\Delta B=385 \mathrm{mT}$. Le calcul, en prenant $P_{\mathrm{I}} P_{\mathrm{R}}=1$ conduit à $T_{1}=1,1 \times 10^{-10} \mathrm{~s}$ et $\tau=1,7 \times 10^{-10} \mathrm{~s}$. Cette dernière valeur est comparable à celles $1-2 \times 10^{-10} \mathrm{~s}$ [5] et $3 \times 10^{-10} \mathrm{~s}$ [23] trouvées par d'autres auteurs dans des super réseaux $\mathrm{GaAs} / \mathrm{AlAs}$ ayant des caractéristiques proches du nôtre.

Pour une excitation résonnante à partir de la sousbande des trous légers, nous avons vu que la valeur de $\mathfrak{T}$ passait par un minimum proche de zéro (pic $B$ du SEL). Dans une telle situation le calcul de la polarisation de la luminescence ne se fait plus d'une manière aussi simple que précédemment. Il résulte d'une quasi-compensation entre forte orientation de spin $-1 / 2$ (issue des états de valence lourds) et $+1 / 2$ (issue des états légers). Cet équilibre est facilement détruit par le champ transverse car il est vraisemblable que suivant la valeur $( \pm 1 / 2)$ du spin photocréé, les populations d'électrons n'aient pas les mêmes durées de vie (ils sont générés à des valeurs différentes du vecteur d'onde). Leur évolution, avant recombinaison radiative, peut donc être distincte et rendre ainsi très embrouillée l'analyse des données d'effet Hanle.

Pour terminer cette discussion, il faut noter l'extrême sensibilité des résultats à des contraintes parasites : lorsque la luminescence est étudiée près du point de collage, les énergies caractéristiques du SEL ne sont modifiées que de quelques meV, mais les degrés d'orientation mesurés le sont beaucoup plus. Par exemple, des taux négatifs sont observés dans la région de la raie $B$ (excitons légers). Dans cette même zone, on note aussi l'apparition d'un pic très fortement orienté aussi bien en polarisation circulaire qu'en polarisation linéaire, ce qui « signe » l'existence d'un double effet Raman résonnant [24]. Nous pensons qu'un tel effet apparaît lorsque la séparation entre les sous-bandes de trous légers et de trous lourds (distance entre les pics A et B du SEL) se rapproche de l'énergie du phonon LO dans GaAs (36,7 meV).

\section{Conclusion.}

Nous avons utilisé la technique de pompage optique pour caractériser un super réseau à courte période GaAs/AlAs proche de la limite des composés à bande interdite indirecte. Nous avons déterminé la valeur respective des temps de relaxation de spin $\left(T_{1}\right)$ et de vie $(\tau)$ des électrons photocréés. La présence de puits élargis dans l'échantillon a permis la mise en évidence d'oscillations de relaxation sur les spectres d'excitation de la luminescence et de la polarisation qu'il reste à étudier en détail. La nature directe de l'échantillon semble confirmée par nos expériences, encore que de nombreuses questions restent ouvertes à cet égard, à commencer par les règles régissant la polarisation des transitions « pseudo-directes » associées aux états X. La mise en évidence d'un double effet Raman résonnant quand on déplace le point d'impact du faisceau incident sur la surface de l'échantillon montre que ce type d'expériences dans de telles hétérostructures est très sensible aux effets des contraintes résultant, par exemple, de la fixation de l'échantillon sur le porte objet.

\section{Remerciements.}

Nous sommes heureux de remercier F. Mollot du L2M pour de fructueuses discussions. Nous remercions le groupe de Spectroscopie Raman du LPS pour le prêt du cryostat à circulation d'hélium. 


\section{Bibliographie}

[1] Minami F., Hirata K., Era K., Yao T. et Masuмото Y., Phys. Rev. B 36 (1987) 2875.

[2] Moore K. J., Dawson P. et Foxon C. T., J. Phys. France 48 (1987) C5-525.

[3] Danan G., Etienne B., Mollot F., Planel R., Jean-Louis A. M., Alexandre F., Jusserand B., Leroux G., Marzin J. Y., SAVARY H. et Sermage B., Phys. Rev. B 35 (1987) 6207.

[4] Danan G., Ladan F. R., Mollot F. et Planel R., Appl. Phys. Lett. 51 (1987) 1605.

[5] Finkman E., Sturge M. D., Meynadier M. H., NAHORY R. E., TAMARgo M. C., HWANG D. M. et Chang C. C., J. Luminescence 39 (1987) 57.

[6] Danan G., Thèse, Université Paris VI (1988).

[7] Bastard G., Phys. Rev. B 24 (1981) 5693.

[8] Miller R. C. et Kleinman D. A., J. Luminescence 30 (1985) 520.

[9] Miller R. C., Kleinman D. A., Nordland Jr. W. A. et Gossard A. C., Phys. Rev. B 22 (1980) 863.

[10] Weisbuch C., Miller R. C., Dingle R., Gossard A. C. et Wiegmann W., Solid State Commun. 37 (1981) 219.

[11] Moore K. J., Dawson P. et Foxon C. T., Phys. Rev. B 34 (1986) 6022.

[12] Lampel G., Phys. Rev. Lett. 20 (1968) 491.
[13] Hermann C., Lampel G. et Safarov V. I., Ann. Phys. Fr. 10 (1985) 313.

[14] Twardowski A. et Hermann C., J. Phys. 48 (1987) C5-211.

[15] Bacquet G., Bandet J., Fabre F., Frandon J. et Paget D., J. Appl. Phys. 58 (1985) 3541.

[16] Paget D., Lampel G., Sapoval B. et Safarov V. I., Phys. Rev. B 15 (1977) 5780.

[17] Jiang D. S., Kelting K., Isu T., QueIsSeR H. J. et Ploog K., J. Appl. Phys. 63 (1988) 845.

[18] D'Yakonov M. I. et Perel' V. I., Zh. Eksp. Teor. Fiz. 60 (1971) 1954 [Soviet. Phys. JETP 33 (1971) 1053].

[19] Nakamura A. et Weisbuch C., Solid State Commun. 32 (1979) 301.

[20] Deveaud B., Chomette A., Lambert R., REgreny A., Romestain R. et Edel P., Solid State Commun. 57 (1986) 885.

[21] Bonnot A., Planel R. et Benoit à la Guillaume C., Phys. Rev. B 9 (1974) 690.

[22] Weisbuch C. et Hermann C., Phys. Rev. B 15 (1977) 816.

[23] Lavallard P., Communication privée.

[24] Planel R. et Benoit à la Guillaume C., Optical Orientation, Modern Problems in Condensed Matter Science, Eds. F. Meier et B. P. Zakharchenya (North Holland, Amsterdam) 1984, Vol. 8 . 